International Journal of Advanced Trends in Computer Science and Engineering

Available Online at http://www.warse.org/IJATCSE/static/pdf/file/ijatcse1181022021.pdf

https://doi.org/10.30534/ijatcse/2021/1181022021

\title{
Covid-19 Mask Detection
}

\author{
Umme Habeeba $\mathbf{R}^{1}$, Zuveriya ${ }^{2}$, K B Drakshayini ${ }^{3}$ \\ ${ }^{1}$ Student, Department of Information Science and Engineering, VVIET Mysore, India, alizahriaz99@gmail.com \\ ${ }^{2}$ Student, Department of Information Science and Engineering, VVIET Mysore, India, zuvizr@gmail.com \\ ${ }^{3}$ Faculty, Department of Information Science and Engineering, VVIET, Mysuru,India, drakshakb@gmail.com
}

\begin{abstract}
The corona epidemic poses a global health problem and therefore effective preventive measures are worn in public places, according to the World Health Organization (WHO). The COVID-19 epidemic has forced governments around the world to impose restrictions on the transmission of the virus. Reports show that wearing the right face while in public places and at work clearly reduces the risk of transmission. An effective and economical way to use machine learning is to create a safe environment for device setup. A hybrid model using the depth of the face mask detection machine will be introduced. The face mask detection database contains a mask and in addition to the facial images, we will use OpenCV to perform real-time facial detection from live streaming via our webcam. We will use the database to create a COVID-19 face mask detector from a computer view using Python, OpenCV, and Tensor Flow and Cameras. We aim to determine whether the person in the picture/video is wearing a face mask or not with the help of computer vision and in-depth reading and to show the same with caution. Steps to modeling are data collection, pre-processing, data classification, model testing, and modeling.
\end{abstract}

Key words: Covid-19, Mask Detection, Machine Learning, OpenCV, TensorFlow, Keras.

\section{INTRODUCTION}

The practice of wearing masks in public is growing as a result of the COVID-19 coronavirus epidemic worldwide. Scientists have confirmed that wearing face masks is effective in preventing the transmission of COVID-19. [2] COVID19 (also known as the coronavirus) is the latest pandemic that has plagued human health in the last century. By 2020, the rapid spread of COVID-19 has forced the World Health Organization to declare COVID-19 a global epidemic. More than five million cases have been infected with COVID-19 in less than 6 months in 188 countries. However, some problems are faced by the authorities while employing a large number of people with a different practice. Authorities need a solution to regulate the implementation of legislation, which begins with the immediate and accurate availability of data. One of the solutions is to use automatic facial recognition in the region to distinguish between people who wear a mask and those who do not. The virus spreads in close proximity to densely populated and overcrowded areas. The corona epidemic has raised an unprecedented level of scientific cooperation worldwide. Artificial Intelligence (AI) based on machine learning and in-depth learning can help combat Covid-19 in many ways. Mechanical studies allow researchers and medical professionals to examine large amounts of data to predict the distribution of COVID-19, to serve as an early warning system for potential diseases, and to identify vulnerable people. People are forced by law to wear public masks in all countries. These laws and regulations were developed as an act of growing growth in conditions and deaths in many places. However, the process of monitoring large groups of people becomes more complicated. The precautionary measures include the detection of anyone who does not wear a face mask. Here we present a face-to-face acquisition model based on computer vision and in-depth learning. The proposed model can be integrated with surveillance cameras to prevent the transmission of COVID-19 by allowing the detection of people wearing masks who do not wear face masks. Model integration between in-depth learning and traditional methods of machine learning with OpenCV, robust flow, and Cameras. We used the in-depth transmission of the feature extractor and integrated it with three machine learning algorithms. We introduced comparisons between them to find the most suitable algorithm that gained the highest accuracy and spent less time on the training and acquisition process. The model built into this study could be performed on surveillance cameras to prevent the transmission of COVID-19 transmission by detecting non-wearing masks [5].

\subsection{Machine Learning}

Machine learning (ML) is the study of computer algorithms that develop automatically through experience. It looks like a 
subset of AI. Machine learning algorithms create a sample data model that is supported, called "training data",in order to create predictions or decisions without explicit planning to try to do so. Machine learning algorithms are used for good types of programs, such as email filtering and computer vision, where it is difficult or impossible to develop general skills to perform the required tasks. Machine learning is closely associated with computational calculations, which focus on computer-generated forecasts. The study of the application of mathematics brings methods, teaching, and application backgrounds into the field of machine learning.[2]Data processing can be a related study field, which focuses on data analysis by unchecked reading. In its application to business problems, machine learning is further referred to as predictive statistics.

\subsection{OpenCV}

OpenCV (Open-Source Computer Vision Library) is an open-source computer vision and machine learning software library. OpenCV was built to supply a standard infrastructure for computer vision applications and to accelerate the utilization of machine perception within commercial products. Being a BSD-licensed product, OpenCV makes it easy for businesses to utilize and modify the code. The library has quite 2500 optimized algorithms, which incorporates a comprehensive set of both classic and state-of-the-art computer vision and machine learning algorithms. These algorithms are often wont to detect and recognize faces, identify objects, classify human actions in videos, track camera movements, track moving objects, extract 3D models of objects, produce 3D point clouds from stereo cameras, stitch images together to supply a high-resolution image of a whole scene, find similar images from a picture database, remove red eyes from images taken using flash, follow eye movements, recognize scenery and establish markers to overlay it with augmented reality, etc. The library is employed extensively in companies, research groups, and by governmental bodies. There are over 500 algorithms and about 10 times as many functions that compose or support those algorithms. OpenCV is written natively in $\mathrm{C}++$ and features a template interface that works seamlessly with STL containers [2].

\subsection{TensorFlow}

TensorFlow may be a free and open-source software library for dataflow and differentiable programming across a variety of tasks. it's a symbolic math library and is additionally used for machine learning applications like neural networks. It is used for both research and production at Google, TensorFlow is Google Brain's second-generation system. Version 1.0.0 was released on February 11, While the reference implementation runs on single devices, TensorFlow can run on multiple CPUs and GPUs. Tensor Flow is out there on 64-bit Linux, macOS, Windows, and mobile computing platforms including Android and iOS. Its flexible architecture allows for the straightforward deployment of computation across a spread of platforms (CPUs, GPUs, TPUs), and from desktops to clusters of servers to mobile and edge devices. The name Tensor Flow derives from the operations that such neural networks perform on multidimensional data arrays, which are mentioned as tensors. During the Google I/O Conference in June 2016, Jeff Dean stated that 1,500 repositories on GitHub mentioned TensorFlow, of which only 5 were from Google. Unlike other numerical libraries intended to be used in Deep Learning like Theano, TensorFlow was designed to be used both in research and development and in production systems. It can run on a single CPU[2].

\subsection{Keras}

Keras is an API designed for the citizenry, not machines. Keras follows best practices for reducing cognitive load: it offers consistent $\&$ simple APIs, it minimizes the number of user actions required for common use cases, and it provides clear \& actionable error messages. It also has extensive documentation and developer guides. Keras contains numerous implementations of commonly used neural network building blocks like layers, objectives, activation functions, optimizers, and a number of tools to form working with image and text data easier to simplify the coding necessary for writing deep neural network code. The code is hosted on GitHub, and community support forums include the GitHub issues page and a Slack channel. Keras may be a minimalist Python library for deep learning which will run on top of Theano or Tensor Flow. it had been developed to form implementing deep learning models as fast and straightforward as possible for research and development. It runs on Python 2.7 or 3.5 and may seamlessly execute on GPUs and CPUs were given the underlying frameworks. it's released under the permissive MIT license. [2]

\section{RELATED WORK}

ToshanlalMeenpal, Ashutosh Balakrishnan, Amit Verma, "Facial Mask Detection using Semantic Segmentation" Initially they have proposed a way of obtaining segmentation masks directly from the pictures containing one or more faces in several orientation. The input image of any arbitrary size is resized to $224 \times 224 \times 3$ and fed to the FCN network for feature extraction and prediction. The output of the network is then subjected to post processing [3].Initially the pixel values of the face and background are subjected to global thresholding. then is skilled median filter to get rid of the high frequency noise then subjected to Closing operation to fill the 
gaps within the segmented area. After this bounding box is drawn round the segmented area. Post processing on the anticipated mask obtained is performed in order that the irregularities within the region are often filled and to get rid of the unwanted errors. This they need performed by first passing the mask through Median filter then performing the Closing Operation [3].

The disadvantages the following work is that it detects only static images, its accuracy is less and the time taken for predicting is more making it less efficient.

Vinitha, Velantina, "Covid-19 facemask detection with deep learning and computer vision" they have used Caffee and MxNet as a deep learning framework for their model for face mask detection.

Samuel Ady Sanjaya, Suryo Adi Rakhmawan, "Face Mask Detection Using MobileNetV2 in The Era of COVID-19 Pandemic" in this related paper their accuracy was fluctuating in each epoch stage resulting in less accuracy.

\section{Proposed SYSTEM}

The mask recognition during this study is developed with a machine learning algorithm through the image classification method: MobileNetv2. MobileNetV2 may be a method supported Convolutional Neural Network (CNN) that developed by Google with improved performance and enhancement to be more efficient. There has been a lot of discussion around deep learning-based approaches for person detection. This encouraged us to come up with our own algorithm to solve this problem.

Our work on face mask detection comprises of data collection to tackle the variance in kinds of face masks worn by the workers. The face mask detection model is a combination of face detection model to identify the existing faces from camera feeds and then running those faces through a mask detection model.

The proposed system focuses on the way to identify the person on image/video stream wearing a mask with the assistance of computer vision and deep learning algorithm by using the OpenCV, Tensor flow, Keras, and PyTorch library. Figure 1 shows the 2 approaches and steps in detecting face mask:

1. Train Deep learning model (MobileNetV2)

2. Apply mask detector over images / live video stream

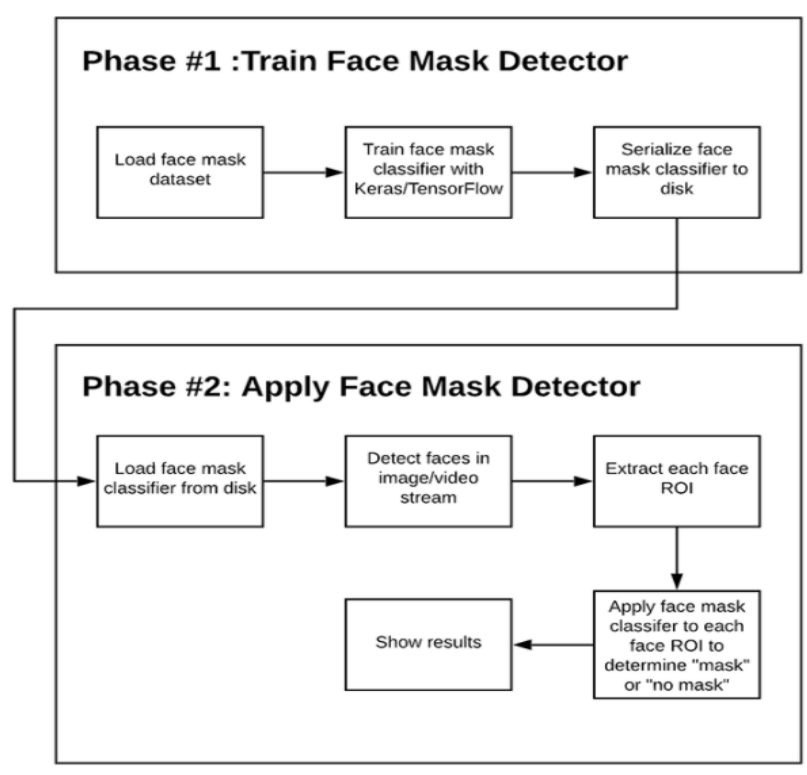

Figure 1:Phases in building a COVID-19 face mask detector

\section{ARCHITECTURE}

The below Figure 2 shows the model produced by the following steps (1) data collecting, (2) pre-processing, (3) split the info, (4) building the model, (5) testing the model, and eventually (6) implement the model. [1]

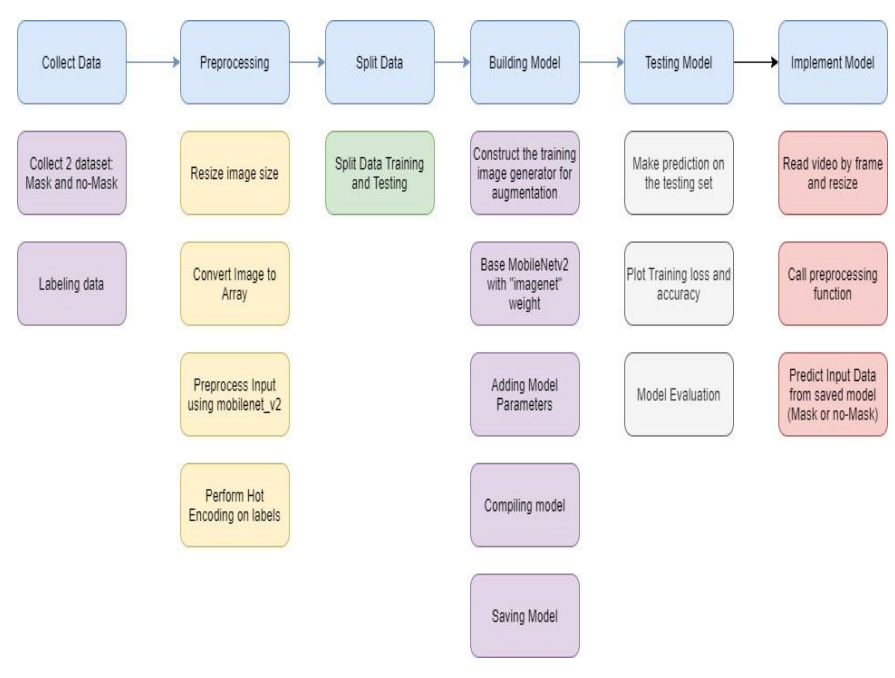

Figure 2: Steps in building the model

1. Data Collecting: the event of the mask Recognition model begins with collecting the info. The dataset trains data on people that use masks and who don't. The model will differentiate between people wearing masks and not. For building the model, this study uses 1.916 data with mask and 1.930 data without a mask. [1],[4]At this step, 
the image is cropped until the sole visible object is that the face of the thing. the subsequent step is to label the info. the info which has been collected is labeled into two groups; with and without a mask as shown in the Figure 3. After the info has been labeled, it's grouped into those two groups.

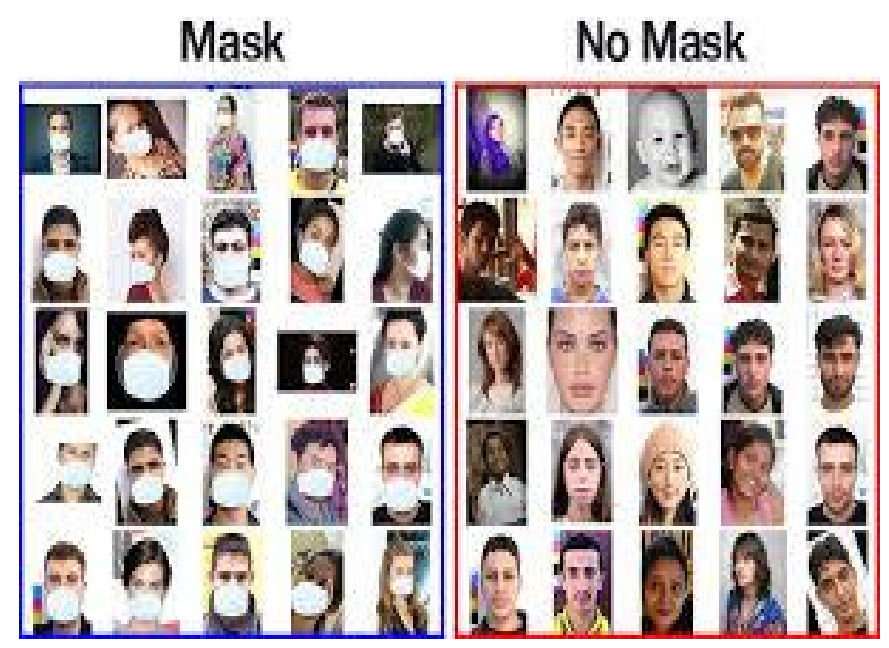

Figure 3: Depiction of 2 datasets to be trained

2. The pre-processing phase may be a phase before the training and testing of the info. There are four steps within the pre-processing which are resizing image size, converting the image to the array, pre-processing input using MobileNetV2, and therefore the last is performing hot encoding on labelsas shown in the Figure 4. The resizing image may be a critical pre-processing step in computer vision thanks to the effectiveness of coaching models. The smaller size of the image, the higher the model will run. during this study, resizing a picture is making the image into $224 \times 224$ pixels [8]. the subsequent step is to process all the pictures within the dataset into an array. The image is converted into the array for calling them by the loop function. then, the image is going to be wont to pre-process input using MobileNetV2. and therefore, the last step during this phase is performing hot encoding on labels because many machine learning algorithms cannot operate data labeling directly. They require all input variables and output variables to be numeric, including this algorithm[1]. The labeled data are going to be transformed into a numerical label;therefore, the algorithm can understand and process the info.

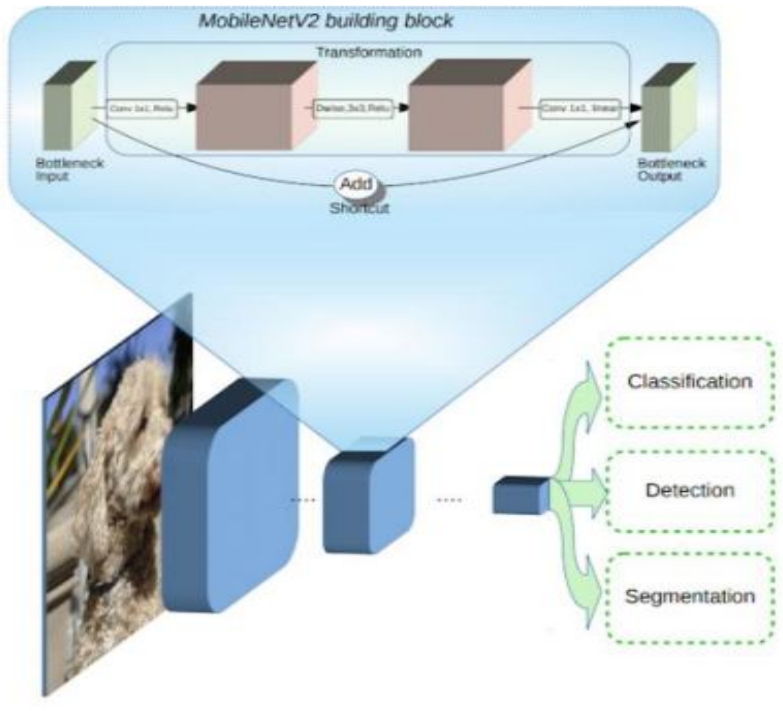

Figure 4: The Preprocessing phase using MobileNetV2

3. Split the info. After the pre-processing phase, the info is split into two batches, which are training data namely 75 percent, and therefore the rest is testing data.[1] Each batch is containing both with-mask and without-mask images [6].

4. Building the Model. the subsequent phase is building the model. There are six steps in building the model which is constructing the training image generator for augmentation,the bottom model with MobileNetV2, adding model parameters, compiling the model, training the model, and therefore the last is saving the model for the longer-term prediction process [1].

5. Test Model. To ensure that the model can accurately predict, there are steps to test the model. The first step is to make a prediction on a test set. The result of 20 repetitions in looking at loss and accuracy when training a model [1].

6. Implementing the model: the model is implemented within the video[7].The video reads from frame to border, then the face detection algorithm works. Once the face is detected, proceed to the next process. From the found frames that contain faces, re-processing will be done including resizing the image size, converting to the same members, processing inputs using MobileNetV2 [9][1].The next step is to predict the input data from the stored model. Predict the input image used using the pre-built model. Alternatively, a video frame will also record whether or not a person wears a mask and a percentage of speculation as shown in the Figure 5. 


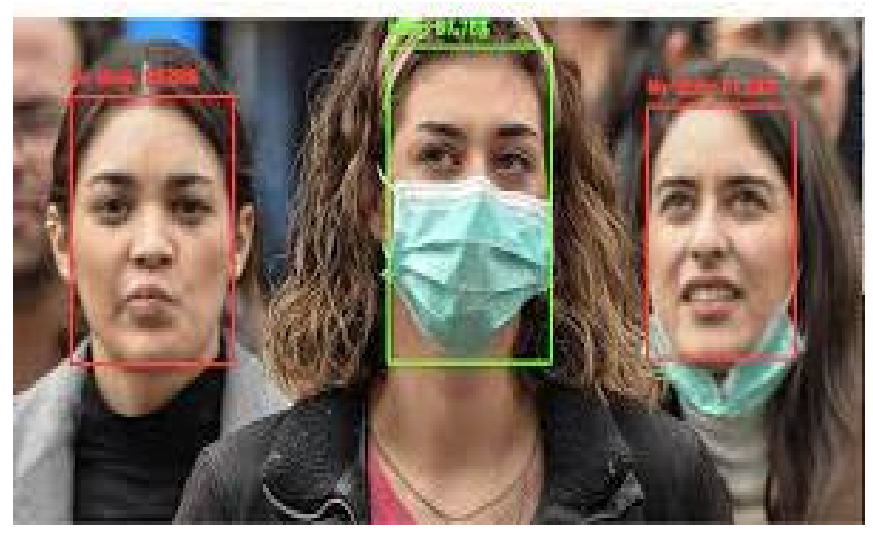

Figure 5: The output of the video frame detecting face mask

\section{CONCLUSION}

In conclusion, this study presents a model that uses machine learning to detect facial mask detection. After the training, verification, and testing phase, the model can provide a percentage of people using face masks in public places with high accuracy. Models tested with real-time photos and video streams. It can be used for a variety of applications. Wearing a mask may be mandatory in the near future, given the Covid-19 problem. Many social service providers will ask customers to wear masks properly to access their services.

The model used will contribute significantly to the public health care system.In the name of a statistical organization that needs to move faster to adopt and take advantage of new digital learning tools and resources, this study could be an easy step for authorities to use more informal data sources to reduce data-dependent, testing, prevention, and planning action against COVID-19.

\section{REFERENCES}

[1] Samuel Ady Sanjaya, Suryo Adi Rakhmawan, "Face Mask Detection Using MobileNetV2 in The Era of COVID-19 Pandemic", April 2020

[2] Vinitha,Velantina, "Covid-19 facemask detection with deep learning and computer vision", August 2020.

[3] ToshanlalMeenpal, Ashutosh Balakrishnan, Amit Verma, "Facial Mask Detection using Semantic Segmentation", October 2019.

[4] Z. Wang, G. Wang, B. Huang, Z. Xiong, Q. Hong, H.Wu, P. Yi, K. Jiang, N. Wang, Y. Peietal., "Masked face recognition dataset and application," arXivpreprint arXiv:2003.09093, 2020.[10]Z.-Q. Zhao, P.Zheng, S.-t. $\mathrm{Xu}$, and $\mathrm{X} . \mathrm{Wu}$, "Object detection withdeep learning: A review,"IEEE transactions onneural networks and learning systems, vol. 30, no.11, pp. 3212-3232, 2019.

[5] Loey, M., Manogaran, G., Taha, M. H. N., \& Khalifa, N. E. M. "A hybrid deep transfer learning model with machine learning methods for face mask detection in the era of the COVID-19 pandemic". Measurement: Journal of the International Measurement Confederation, 2020, doi: 10.1016/j.measurement.2020.108288

[6] Ejaz, M. S., Islam, M. R., Sifatullah, M., \& Sarker, A., "Implementation of Principal Component Analysis on Masked and Non-masked Face Recognition”. 2019 1st International Conference on Advances in Science, Engineering and Robotics Technology (ICASERT), 1-5.

[7] Hussain, S. A., \& Al Balushi, A. S. A. "A real time face emotion classification and recognition using deep learning model". Journal of Physics: Conference Series, 1432(1), 12087, 2020.

[8] Qin, B., \& Li, D. "Identifying Facemask-wearing Condition Using Image Super-Resolution with Classification Network to Prevent COVID-19”, 2020.

[9] Sandler, M., Howard, A., Zhu, M., Zhmoginov, A., \& Chen, L. C. "Mobilenetv2: The next generation of on-device computer vision networks", 2020. 